\title{
Stefan Kühl
}

\section{Regelabweichungen zum Schaden und Nutzen der Organisationen}

\section{Zur Funktionalität informaler Belohnungssysteme in Organisationen}

https://doi.org/10.1515/arbeit-2020-0004

Zusammenfassung: In der Forschung über Devianz wird in der Regel davon ausgegangen, dass eigennützige Regelabweichungen von Organisationsmitgliedern für die Organisation schädlich sind. Dabei wird eine Differenz zu für die Organisation funktionalen Regelabweichungen markiert. Basierend auf der Sekundäranalyse zentraler Studien über Unterschlagungen, Korruption oder Abstinenz von Organisationsmitgliedern wird in diesem Beitrag nach einer möglichen Funktionalität dieser Devianz für die Organisation gefragt. Während es sich in vielen Fällen um eine Schädigung von Organisation handelt, kann es - so das Argument - Fälle geben, in denen die Duldung von Unterschlagung, Korruption und Abstinenz als Teil eines informalen Belohnungs- und Anreizsystems genutzt wird.

Schlüsselwörter: Devianz, Arbeitsorganisationen, informale Belohnung, Leistungsmotivation

\section{Deviation from rules to the detriment or benefit of organizations}

The functionality of informal reward systems in organizations

\begin{abstract}
Deviance research usually proceeds on the assumption that selfserving deviations from organizational or legal rules are detrimental to the organization. This assumption suggests a distinction against deviations that are functional for the organization. Based on a secondary analysis of seminal studies
\end{abstract}

Prof. Dr. Stefan Kühl, Professur für Organisationssoziologie, Universität Bielefeld, Universitätsstraße 25, 33615 Bielefeld, Deutschland. E-Mail: stefan.kuehl@uni-bielefeld.de 
about embezzlement, corruption or abstinence from work, this paper examines possible functionalities of these deviant practices for the organization. Whereas they are, in many cases, detrimental to the organization, the author argues that in other cases toleration of embezzlement, corruption and abstinence from work may serve as part of a system of informal reward or incentive.

Keywords: deviance, work organizations, informal reward, perfromance motivation

\section{Einleitung}

Irgendjemand profitiert immer von der Abweichung von Regeln oder Gesetzen. Bei brauchbarer Illegalität und funktionalen Regelabweichungen hat die Organisation Vorteile. Das streng genommen verbotene, aber in der Praxis nicht verfolgte Hinterziehen von Steuern durch Internetkonzerne, das bestenfalls halblegale Ausplündern von Kunden durch Banken oder die Sprengstoffanschläge durch terroristische Gruppen mögen für die Bürger eines Staates, die Kunden einer Bank oder die Opfer eines Anschlages Nachteile mit sich bringen, den Organisationen verschafft all dies aber erst einmal unmittelbare Vorteile. In vielen Fällen ziehen jedoch auch einzelne Organisationsmitglieder persönliche Vorteile aus illegalem Verhalten. Schließlich kann das Hinterziehen von Steuern, das Ausrauben von Bankkunden oder ein religiös motivierter Selbstmordanschlag dem Steuerhinterzieher, Räuber oder Attentäter unmittelbare Vorteile in Form von Steuerersparnissen, Geldeinnahmen oder im Paradies vorzufindenden Jungfrauen bringen. ${ }^{1}$

In Organisationen wird deswegen sehr genau beobachtet, wem die Regelabweichungen und Gesetzesbrüche zugutekommen. ${ }^{2}$ Schließlich macht es für Organisationen einen grundlegenden Unterschied, ob von einer Steuerhinterziehung vorrangig sie selbst oder im Gegenteil eher das Organisationsmitglied profitiert, ob die Bank oder die Bankkunden ausgeplündert werden oder ob Mitglieder oder Gegner der Organisation einem Sprengstoffanschlag zum Opfer fallen. In dem einen Fall handelt es sich um Regelabweichungen, die von Organisationsmitgliedern zum Wohl der Organisation vorgenommen werden, um die Handlungen von „honest crooks“ - ehrlichen Gaunern -, die wegen der Verletzung orga-

\footnotetext{
1 Zur komplexen, aber letztlich eigennützigen Motivlage von Selbstmordattentätern siehe Gambetta (2006).

$2 \mathrm{Zu}$ dieser Unterscheidung siehe nur beispielhaft Michalowski (1985).
} 
nisationseigener Regeln zumindest die Kündigung riskieren oder bei Verstößen gegen Gesetze sogar strafrechtliche Verfolgung. Im anderen Fall handelt es sich um Regelabweichungen, bei denen Mitarbeiter aus selbstsüchtigen Motiven der Organisation schaden (so z.B. Punch 1996, 2).

Nachdem in der Forschung über Regelabweichungen mit dem Zentralbegriff der White-Collar-Kriminalität die Frage, wem dies nützt, weitgehend undifferenziert betrachtet wurde, wird in der Forschung über Illegalitäten in Organisationen zunehmend genauer beobachtet, wer davon profitiert (so prominent Coleman 1987, 406). ${ }^{3}$ Es wird zwischen „Corporate Crimes“, Gesetzesverstößen zum Nutzen der Organisation, und „Occupational Crimes“, Regelabweichungen, die lediglich einzelnen Organisationsmitgliedern zugutekommen, unterschieden (Clinard/Quinney 1973). Bei Verwaltungen werden „State-organized Crimes“, also Fälle von für den Staat brauchbarer Illegalität, von Gesetzesverstößen unterschieden, bei denen Staatsbedienstete ihre Positionen für eigene Vorteile ausnutzen (siehe Friedrichs 2010). Bei der Polizei werden Regelabweichungen, die dazu dienen, den Job besser zu machen, von Verstößen, die der persönlichen Bereicherung von Polizisten dienen, abgegrenzt (für einen Überblick siehe Barker/ Carter 1994).

Bei vielen Regelabweichungen ist scheinbar auf den ersten Blick klar, ob es darum geht, sich auf Kosten der Organisation einen eigenen privaten Nutzen zu verschaffen, oder ob es sich um eine Regelabweichung zum Wohl der Organisation handelt. Wenn Manager Banken benutzen, um Sparer um ihre Geldanlagen zu bringen (siehe dazu Calavita/Pontell 1993), oder Politiker mit überhöhten Einkaufspreisen und fiktiven Rechnungen eine Stadt finanziell ausnehmen, fällt es schwer, den Nutzen für die Sparkassen oder die Stadtverwaltung zu erkennen (siehe z.B. Fallstudien in Miller 1992). Wenn Manager Banken dadurch zu retten versuchen, dass sie Bilanzen manipulieren, oder Politiker mit großzügigen Interpretationen des Baurechts ein Großprojekt fristgerecht fertigstellen wollen, kann man erst einmal davon ausgehen, dass es um Regelabweichungen geht, die der Organisation Vorteile verschaffen sollen.

Aber auf den zweiten Blick ist es häufig nicht so klar, welche Rolle der Eigennutz bei einer Regelabweichung spielt. Wie sind Schmiergeldzahlungen durch die Mitarbeiter großer Elektronikkonzerne einzuschätzen, bei denen die Mitarbeiter zwar keine Gelder abgezweigt haben, aber aufgrund ihrer Vertriebserfolge

3 Siehe für die weitgehende Ignorierung des Unterschieds zum Beispiel Sutherland (1949). Dies findet sich auch noch später in der Literatur; siehe nur Mars $(2013,48)$, in der (nur) für die Organisation funktionale Regelabweichung im Flugzeugbau in der Studie von Bensman/Gerver (1963) mit Illegalitäten gleichgesetzt wird, die nur den Organisationsmitgliedern nutzen. 
deutlich höhere Prämienzahlungen durch die Organisation erhalten? Wie sollte man Insiderhandel von Banken beurteilen, bei dem zwar erst einmal die Bank auf Kosten von anderen Anlegern profitiert, aber auch die Händler der Bank ihren Schnitt machen? Wie sieht es aus, wenn Organisationen stillschweigend auf die Attraktivität ihrer jungen weiblichen und männlichen Mitarbeiter setzen und zudem erwarten, dass diese auch anzügliche Bemerkungen oder Berührungen erdulden? Wie muss man Fälle einschätzen, in denen ein Vizepräsident eines Staates, der vorher Vorstandsvorsitzender eines Energiekonzerns gewesen ist, seine Position in der Politik ausnutzt, um die Energiepolitik der Regierung „konzernfreundlicher“ werden zu lassen ${ }^{4}$ Gerade die Grauzonen zwischen für die Organisation brauchbarer und unbrauchbarer Illegalität sind interessant.

Das Ziel dieses Beitrags ist es, den Graubereich zwischen brauchbarer Illegalität (die für die Organisation funktionalen Regelabweichungen) und eigennütziger Illegalität (die nur für das Organisationsmitglied nützlichen Regelabweichungen) näher auszuleuchten. Damit soll eine Forschungslücke in der Organisationswissenschaft geschlossen werden. In der Organisationswissenschaft wird zwar inzwischen der grundlegende Unterschied zwischen diesen beiden Formen von Regelabweichungen erkannt, man interessiert sich aber bisher nicht systematisch dafür, welche Funktionen eigennützige Illegalitäten für die Organisation haben könnten.

Im folgenden Abschnitt werden die verschiedenen Formen eigennütziger Illegalität beleuchtet und deren Verbreitungsgrad diskutiert. Im dritten Abschnitt wird nach möglichen Funktionen eigennütziger Illegalität gefragt. Es wird dabei herausgestellt, dass die Duldung illegaler Belohnungssysteme eine Lösung für das in Organisationen zentrale Motivationsproblem sein kann. Im vierten Abschnitt wird genauer analysiert, weshalb sich informale Belohnungssysteme ausbilden und worin ihre Vorteile für die Organisation liegen können. Im fünften Abschnitt wird gezeigt, wie die Grenzen für die informalen Belohnungssysteme in Organisationen einreguliert werden und welche Rolle Kollegen, Vorgesetzte und Untergebene dabei spielen. Der abschließende sechste Abschnitt diskutiert kurz die Reaktionsformen von Organisationen auf eigennützige Illegalitäten ihrer Mitglieder.

Als Empirie für diesen Artikel dienen die zentralen ethnografischen Studien, die in den letzten fünfzig Jahren zu eigennützigen Illegalitäten erschienen sind. Dabei wurden die empirischen Artikel herangezogen, die in der Diskussion be-

4 Ein interessanter Fall ist die Einschätzung der Politik von Dick Cheney, des ehemaligen Vorstandsvorsitzenden von Halliburton, in seiner Rolle als US-Vizepräsident (Van Natta/Banerjee 2002 nach Friedrichs 2010). 
sonders häufig zitiert werden. In diesen Arbeiten findet sich anfangs noch ein Blick für die Funktionalität informaler Belohnungssysteme für die Organisationen, der aber immer stärker zugunsten eines Blicks auf die Möglichkeiten zu deren Unterbindung aufgegeben wurde. Die im Rahmen dieser Sekundäranalyse herangezogenen ethnografischen Studien können hier nicht umfassend dargestellt werden, sondern werden exemplarisch herangezogen, um mögliche Funktionalitäten für die Duldung der Regelabweichungen zu analysieren.

\section{Auf der Suche nach persönlichen Vorteilen - Unterschlagung, Korruption und Arbeitsverweigerung}

In Organisationen ist man sich schnell einig, welche Regelabweichungen für Organisationen unbrauchbar sind - nämlich solche, die Mitarbeiter begehen, um sich auf Kosten der Organisation in verbotener Art und Weise persönliche Vorteile zu verschaffen. Man denke an das Stehlen von Kitteln durch Krankenschwestern und Pfleger, um daraus zu Hause Shorts oder Putzlappen zu machen (Dabney 1995, 320). Man erinnere sich an die Tricks, mit denen Stewardessen und Stewards aus den Flugzeugen verbotenerweise Getränke und Essen mitnehmen, indem sie sich den Kauf von Kollegen bescheinigen lassen und die Bescheinigung dann zerreißen, wenn sie beim Verlassen des Flughafens nicht kontrolliert werden. Oder man schaue sich die Raffinesse an, mit denen es Blackjack-Dealern in Kasinos gelingt, trotz strikter Regeln des Umgangs mit Kunden und trotz genauer Videoüberwachung Spieler, die großzügige Trinkgelder geben, zu bevorteilen (Sallaz 2009).

\subsection{Verschiedene Möglichkeiten, Vorteile aus einer organisatorischen Tätigkeit zu ziehen}

Regelabweichungen zum eigenen Vorteil der Organisationsmitglieder werden in dieser Forschung als „Verbrechen gegen die Bürokratie“ bezeichnet (Smigel/ Ross 1970). Es wird zugestanden, dass Organisationen in Form mafiöser Vereinigungen, terroristischer Gruppierungen, marodierender Armeen oder korrupter Sportverbände selbst zu Tätern werden können, aber es wird herausgestellt, dass viele Illegalitäten der Organisationsmitglieder auf Kosten der Organisation gehen. 
Die betrogene oder beraubte Organisation wird selbst zum Opfer von Illegalität, kann aber - anders als betrogene oder beraubte Personen - nicht auf Mitleid der allgemeinen Öffentlichkeit hoffen (Smigel/Ross 1970, 4 f.). Im Gegenteil häufig entstehen Sympathien für Personen, denen es gelingt, der mächtigen Großorganisation ein Schnippchen zu schlagen und auf deren Kosten einen Vorteil zu erzielen. Man muss sich nur die Literatur mit Figuren wie dem Hauptmann von Köpenick (Zuckmayer 2004), dem braven Soldaten Schwejk (Hašek 1960) oder dem vermeintlichen Pan-Am-Piloten, falschen Oberarzt und Scheckbetrüger Frank Abagnale (Abagnale/Redding 2000) ansehen. ${ }^{5}$

Es gibt verschiedene Möglichkeiten, sich illegale Vorteile $\mathrm{zu}$ verschaffen. Eine erste Möglichkeit besteht in der Unterschlagung von Material der Organisation. Das kann die verbotene Nutzung von Telefonen, Kopierern oder Werkzeugen für private Zwecke sein oder auch der illegale Verkauf von Materialien zum Zwecke der persönlichen Bereicherung. ${ }^{6}$ Die Dimensionen solcher Unterschlagungen können von dem verbotenen Aufessen eines Brötchens im Restaurant durch das Service-Personal über das Betreiben eines privaten Zweitgeschäfts mit Ressourcen der Organisation bis hin zum Verkauf ganzer Waffensysteme von Armeeangehörigen auf dem Schwarzmarkt gehen (siehe zum Entwenden in Organisationen z. B. Tucker 1989). ${ }^{7}$

Eine zweite Möglichkeit ist der Einsatz von Korruption zur persönlichen Bereicherung. Hier nutzt ein Organisationsmitglied seine Tätigkeit an der Grenzstelle einer Organisation aus, um sich persönliche Vorteile zu sichern. Dabei kann es sich um den Einkäufer eines Unternehmens handeln, der bei der Vergabe eines Auftrags dafür sorgt, dass „kick backs“ auf sein privates Konto fließen, um Angestellte staatlicher Verwaltungen, die für eine beschleunigte Bewilligung von Sozialhilfe-, Bau- oder Visaanträgen ihnen persönlich zugutekommende Geldzahlungen verlangen, oder um Polizisten, die gegen eine kleinere oder auch größere Gefälligkeit von einer Strafverfolgung absehen. Anders als bei Unterschla-

5 Noch deutlicher als in der Autobiografie von Abagnale wird die Sympathie für die Verbrecher auf Kosten der Großorganisationen noch in der filmischen Umsetzung mit Leonardo DiCaprio in „Catch Me If You Can“. Interessant ist die Differenz zwischen Organisationsmitgliedern, die durch ihr Leiden an der Organisation dazu kommen, dieser ein oder auch mehrere Schnippchen schlagen zu wollen, und Personen, die über Hochstapelei eine Organisationsmitgliedschaft erreichen oder simulieren.

6 Zum Unterschied zwischen der Verwendung für private Zwecke und dem Weiterverkauf siehe Henry/Mars (1978).

7 Bei Hollinger/Clark (1982) wird diese Form der „Property Deviance“ - die Aneignung von „greifbarem Eigentum“ - von der „Production Deviance“ - dem Verstoß gegen formale Arbeitsnormen zur Schonung der eigenen Arbeitskraft - unterschieden. 
gungen, die von einem Organisationsmitglied vorgenommen werden, basiert Korruption immer auf einer Kooperation in Form eines unmoralischen, in vielen Fällen auch verbotenen Tauschs mit einem Organisationsexternen (Neckel 1995). ${ }^{8}$

Eine weitere Variante ist die Schaffung von formal verbotenen Arbeitserleichterungen in der Organisation. Meistens wird beim Einkauf von Arbeitskraft der formale Rahmen festgelegt, in dem sich Mitarbeiter bewegen können: Beginn und Ende der Arbeitszeit, Regeln für Arbeitspausen, Verrichtung von persönlichen Tätigkeiten in der Arbeitszeit oder krankheitsbedingte Abwesenheiten. Vor dem Hintergrund können sich Organisationsmitglieder illegale persönliche Vorteile verschaffen, indem sie verspätet zur Arbeit kommen, früher gehen, die Pausen ausdehnen, die Arbeitszeit für persönliche Aktivitäten wie Börsenspekulationen, Pflege von Internetfreundschaften oder Drogenkonsum nutzen oder krankheitsbedingte Abwesenheiten simulieren. ${ }^{9}$

\subsection{Regelabweichung durch Einzelne, Gruppen von Personen oder alle Organisationsmitglieder}

Die Schaffung illegaler Vorteile auf Kosten von Organisationen kann durch Einzelne stattfinden. In der Literatur werden sie als Falken oder Geier bezeichnet. Bei Falken handelt es sich um Organisationsmitglieder, die häufig ganz selbstverständlich davon ausgehen, dass ihre besondere Leistung durch Belohnungen, notfalls auch am Rande der Legalität, vergütet wird; diese Haltung findet sich nicht selten bei Journalisten, Vertriebsmitarbeitern oder Investmentbankern (siehe dazu Mars 1982, 40 ff.; Mars 2013, 39-40). Bei Geiern handelt es sich um einzelne Organisationsmitglieder, die zwar mit anderen Organisationsmitgliedern kooperieren, um sich an der Organisation zu bereichern, die aber, sobald die „Beute erlegt“ ist, versuchen, sich - auch auf Kosten der Kollegialität - die

8 Sicherlich fällt die Korruption bei „öffentlichen Amtsträgern“ wie beispielsweise Verwaltungsmitarbeitern, Polizisten und Lehrern besonders ins Auge, aber es gibt keinen Grund, Korruption auf den Missbrauch staatlicher Machtpositionen für einen privaten Vorteil zu begrenzen (so noch Senturia 1931). Im Prinzip hat jedes Organisationsmitglied auf einer Grenzstelle die Möglichkeit, den Kontakt zur Umwelt der Organisation zum eigenen Vorteil auszunutzen.

9 Zur Bedeutung siehe Clark/Hollinger (1983). Dieses Phänomen wird im englischen Sprachraum auch unter dem Schlagwort „withdrawal from work hypothesis“, der körperlichen oder mentalen Abwesenheit am Arbeitsplatz aufgrund von Frustration, diskutiert (siehe z.B. Bryant 1975). Im deutschsprachigen Raum wird das Phänomen unter dem Begriff der ,inneren Kündigung behandelt (siehe z. B. Höhn 1986, 1989). 
Beute anzueignen. Es handelt sich dabei häufig um Handwerker, Arbeiter oder Ausfahrer, die die vielfältigen Möglichkeiten, sich kleine oder auch größere Vorteile auf Kosten der Organisation zu verschaffen, ausnutzen (siehe dazu Mars 1982, 108 ff.; Mars 2013, 41).

Häufig finden sich aber auch Gruppen von Personen, die sich dazu verabreden, sich an der Organisation zu bereichern. Es handelt sich dabei um Beutegemeinschaften oder Bereicherungscliquen, die gemeinsam auf Kosten der Organisation persönliche Vorteile erzielen. In der Literatur werden sie als Wolfsrudel bezeichnet. Es handelt sich dabei um Gruppen mit häufig informalen Führern und starker Solidarität untereinander, die bei der Bereicherung auf Kosten der Organisation eng miteinander kooperieren. Man denke nur an die Teams von Müllmännern in der Abfallwirtschaft, die Entladeteams in Häfen und Lagern oder an die Polizisten einer Wache. So war es beispielsweise bei der New Yorker Polizei lange Zeit üblich gewesen, Verhaftungen kurz vor Dienstschluss vorzunehmen und dabei möglichst viele Polizisten hinzuziehen, um finanziell lukrative Überstunden zu generieren (siehe Mars 1982, 89 ff.; Mars 2013, 40). ${ }^{10}$

Es gibt aber auch Organisationen, in denen Praktiken zur Bereicherung auf Kosten der Organisation von fast allen Organisationsmitgliedern betrieben werden. Aus Stahlkonzernen ist bekannt, dass von der Personalabteilung geduldet wird, dass ein informaler Sonderurlaub für Mitarbeiter mit der Bemerkung „krank ohne Krankenschein“ in der Schichtplanung eingetragen wird. In einigen Organisationen ist die informale Abwesenheit von Personal so in die Erwartungshaltung eingebaut, dass die Anwesenheit des gesamten Personals ein Problem für die Organisation darstellt. In der Forschung ist die Rede von „Organisationen korrupter Individuen“ (Pinto u.a. 2008, 688).

\subsection{Regelabweichungen auf verschiedenen Ebenen der Organisation}

Wir wissen, dass sich Regelabweichungen in allen funktionalen Bereichen und auf allen hierarchischen Ebenen von Organisationen finden lassen. Wir finden Abweichungen von Regeln aus Eigennutz in Forschung und Entwicklung, im Ein-

10 Bei Gerald Mars' Aufteilung handelt es sich um ein an Mary Douglas angelehntes Vier-FelderSchema mit den beiden Dimensionen Vernetzungscharakter (grid) und Gruppendruck (group strength). Auf die Darstellung der durch Fatalismus gekennzeichneten Esel, die der Organisation vorrangig durch Frustration schaden, wird hier nicht eingegangen (siehe dazu Mars 1982, 66 ff.; Mars 2013, $41 \mathrm{f}$.). 
und Verkauf, im Produktionsbereich, im Personalbereich und im Qualitätsmanagement. Und wir wissen, dass Regelabweichungen aus Eigennutz bei Spitzenmanagern der obersten Hierarchieebene, beim mittleren Management, bei den Vorarbeitern und Teamleitern der untersten Führungsebene bis hin zu Mitarbeitern der untersten Hierarchieebene vorkommen (siehe dazu Wardi/Weitz 2004, 4).

Auffällig ist, wie stark dieses Phänomen in der Diskussion auseinandergezogen wird, je nachdem, ob die Personen, die Illegalitäten aus Eigennutz begehen, in der Organisation „unten“ oder „oben“ stehen (siehe dazu Horning 1970). Wenn es sich um Personen auf den unteren Hierarchiestufen handelt, ist die Rede von „Kriminalität am Arbeitsplatz“, „Arbeitsplatz-Devianz“ oder „kontraproduktivem Arbeitsverhalten““. ${ }^{11}$ Wenn es sich um Personen der oberen Hierarchiestufen handelt, ist von Verbrechen der „upper world“ (Morris 1934, 153 f.), von „suite crimes“, Kriminalität in Business-Räumen, oder von der ,white collar criminality“, Kriminalität der Personen mit den weißen Hemdkragen, die Rede. ${ }^{12}$

Bei der Begriffswahl wird deutlich, wie stark der Organisationsfokus von einem an Klassenunterschieden ausgerichteten Fokus überlagert wird. ${ }^{13}$ Mit dem Begriff „crimes of the upper world“, „suite crime“ oder „white collar crime“ soll darauf aufmerksam gemacht werden, dass Gesetzesverstöße eben nicht nur von Mitgliedern der verarmten „unteren Klassen“, sondern auch von Mitgliedern der „oberen Klassen“ begangen werden. Deren Regelverstöße würden aber nicht in der gleichen Weise verfolgt, weil Personen aus den oberen Klassen in der Lage seien, sich einer Strafverfolgung zu entziehen, und selbst wenn sie ertappt werden, vor Gericht milder behandelt würden (Sutherland 1983, 5-7). ${ }^{14}$

Die Organisation wird dabei entweder Opfer ihrer Mitglieder, oder die Organisation wird von ihren Mitgliedern benutzt, um sich auf verbotene Weise per-

11 Die Begriffe klingen im Englischen besser. Die Rede ist von „,ounter productive work behaviour“ (siehe z.B. Mangione/Quinn 1975); von „workplace deviance“, von „workplace delinquency“ oder von „white collar crime“, siehe maßgeblich Sutherland (1949).

12 Die Anlehnung an männliche Kleidungsnormen ist dabei nicht zufällig, weil lange Zeit davon ausgegangen wurde, dass vorwiegend Männer hohe Positionen in der Gesellschaft erlangen, um von dort aus kriminelle Aktionen unternehmen zu können.

13 Bei Sutherland $(1983,7)$ wird ,white collar crime“ definiert ,as a crime committed by a person of respectability and high social status in the course of his occupation“.

14 Das Konzept des „white collar crime“ hat die Diskussion in den USA stark geprägt. Das grundlegende Problem dieses Konzepts ist jedoch gewesen, dass es Regelabweichungen an die Zugehörigkeit zu Klassen und nicht zu Organisationen gebunden hat (siehe die frühe Kritik z.B. von Edelhertz 1970 oder Lauderdale u.a. 1978). Zum grundlegenden Unterschied einer über Klassen argumentierenden (impliziten) marxistischen Theorie von einer systemtheoretischen Herangehensweise siehe Kühl (2017). 
sönliche Vorteile zu verschaffen. Statt wie Straßenkriminelle Pistolen oder Messer als Mittel für ihre Verbrechen einzusetzen, nutzen die Verbrecher hier die Organisation als Instrument (Wheeler/Rothman 1982, 1406; nach Punch 1996, 214).

\section{Illegale Lösungen für das Motivationsproblem}

Während man bei einzelnen Regelabweichungen zum Nutzen der Organisation noch teilweise mit Verständnis rechnen kann, findet man für Regelabweichungen, die lediglich dem persönlichen Nutzen dienen, kaum Verständnis. ${ }^{15}$ Man mag bei der einen oder anderen Gelegenheit kleinere Möglichkeiten zur persönlichen Vorteilsnahme in der eigenen Organisation nutzen; werden diese Fälle jedoch bekannt, dann kann man kaum mit sozialer Unterstützung rechnen. Die Rede ist davon, dass die alltäglichen Unterschlagungen, die epidemische Korruption und die systematische Arbeitsverweigerung die Leistungsmoral in der Gesellschaft untergraben (siehe dazu Höffling 2002, 13).

Aber wenn man genau hinschaut, erkennt man, wie in vielen Organisationen akzeptiert wird, dass sich Mitarbeiter auf illegale Weise kleine persönliche Vorteile verschaffen. Man denke nur an die Duldung der eigentlich verbotenen Nutzung von Diensttelefonen, Dienstfahrzeugen oder Dienstwerkzeugen für den privaten Gebrauch, an das weitgehend akzeptierte Nutzen von Dienstkopierern für private Zwecke oder die tolerierte Nutzung des Internets während der Arbeitszeit, um Facebook-Freundschaften zu pflegen, sich über die politische Weltlage zu informieren oder das private Börsenportfolio zu optimieren.

Was sind die Gründe für diese Duldung eigentlich verbotener persönlicher Vorteile? Kann es für Organisationen funktional sein, dass sich Mitarbeiter durch Unterschlagungen, Diebstahl oder Arbeitsentzug persönliche Vorteile verschaffen?

\subsection{Zum Motivationsproblem von Mitarbeitern}

In den meisten Organisationen - man denke nur an Unternehmen, Verwaltungen, Polizeien oder Armeen - wird man nicht Mitglied, weil einem die Zwecke der Organisation so förderungswürdig erscheinen oder die in der Organisation angebotenen Tätigkeiten so attraktiv sind. Die Fließbandmontage von Kühlschrän-

15 Siehe nur als ein Beispiel die an Praktiker gerichtete Literatur zu Diebstählen durch Organisationsmitglieder, siehe unter gleichem Titel Bliss/Aoki (1993) und New (1994). 
ken, der Verkauf von Versicherungspolicen, die Bearbeitung von Asylanträgen oder das Exerzieren auf einem Kasernenhof haben jedenfalls für die meisten Personen keinen so hohen Befriedigungsgehalt, dass sie ohne äußeren Anreiz einen erheblichen Teil ihres Tages mit diesen Tätigkeiten verbringen würden. ${ }^{16}$

Organisationen setzen deswegen ein bewährtes Mittel ein, um Organisationsmitglieder für eher unattraktive Tätigkeiten zu motivieren - Geld. Der Vorteil von Geld liegt in der hohen Flexibilität bei der Herstellung von Motivation (Luhmann 1964, 94 ff.) Durch Geldzahlungen können Mitglieder veranlasst werden, unterschiedliche Tätigkeiten auszuüben unabhängig davon, wie attraktiv deren Ausübung ist. Selbst für Aufgaben wie das Reinigen von ölverseuchten Stränden, das Kopieren von dicken Büchern oder die Bearbeitung von Baugenehmigungen können Personen motiviert werden, vorausgesetzt die Organisation ist bereit, ihre Mitglieder dafür zu bezahlen. ${ }^{17}$

Dabei müssen Mitglieder nicht durch Geldzahlungen für jede Handlung einzeln motiviert werden. Eine Chefin muss ihre Sekretärin nicht für jeden abgestimmten Termin, für jede erstellte Folienpräsentation oder für jeden geschriebenen Brief gesondert entlohnen, sondern sie geht davon aus, dass all diese Tätigkeiten im Rahmen der monatlichen Gehaltszahlung abgegolten werden. Ein Offizier muss seine Soldaten nicht für jede ordentlich geputzte Waffe, jedes korrekt gemachte Bett oder jeden erfolgreich durchgeführten Gewaltmarsch gesondert belohnen, sondern geht davon aus, dass seine Anweisungen befolgt werden, solange die Soldaten Interesse daran haben, in der Armee zu verbleiben. In der Organisationswissenschaft wird dieser Prozess als Generalisierung der Mitgliedschaftsmotivation (Luhmann 1964, 93 ff.) bezeichnet.

Sicherlich - die über Geld geschaffene Indifferenzzone von Mitarbeitern gegenüber organisatorischen Erwartungen kann nicht beliebig ausgeweitet werden. Die meisten Mitarbeiter in der öffentlichen Verwaltung sind nicht bereit, körperliche Gewalt gegen Klienten anzuwenden, die meisten Mitarbeiter in klassischen Produktionsunternehmen würden sich weigern, eine Woche ohne jede Pause durchzuarbeiten, und auch Soldaten sind nicht bereit, alles zu tun, was die Militärführung von ihnen verlangt. Aber in den meisten Fällen ist die Indiffe-

16 Grundlegend zum Problem der Entfremdung von Mitgliedern in Organisationen Blauner (1964).

17 Wenn Geld als Motivationsmittel eingesetzt wird, handelt es sich in der industriesoziologischen Diktion zwangsläufig um Arbeitsorganisationen. Für die Analyse von Organisationen, die andere Motive als Geld zur Mitgliedschaftsbindung einsetzen - man denke zum Beispiel an Vereine - müsste die Interpretation modifiziert werden. Siehe zum Unterschied der Mittel zur Mitgliedschafts- und weitergehend der Teilnahmemotivation Kühl (2011, 37 ff.). 
renzzone der Mitarbeiter ausreichend groß, dass Organisationen Mitarbeiter mit sehr unterschiedlichen Anforderungen konfrontieren können.

Für die Organisation hat das einen großen Vorteil. Man kauft Leistungen die Arbeitskraft ihrer Organisationsmitglieder - pauschal ein und erspart sich dadurch die Notwendigkeit, dass jede einzelne Aktivität erfasst und vergütet werden muss. Man geht einfach davon aus, dass die Mitarbeiter im Rahmen der Lohn- und Gehaltszahlung das erfüllen, was von ihnen erwartet wird.

\subsection{Das Problem der Übersetzung in reale Arbeitsleistungen}

Bei der Motivation von Organisationsmitgliedern mithilfe von Geldzahlungen gibt es jedoch ein Problem. Die Entlohnung von Mitarbeitern stellt erst einmal nur sicher, dass diese zu einer bestimmten Zeit in der Organisation sind. Aber die Organisation kann sich nicht sicher sein, was die Mitarbeiter in dieser Zeit machen. Anders als bei erworbenen Maschinen kann die Organisation bei eingekaufter Arbeitskraft nicht davon ausgehen, dass diese sich reibungslos in den Arbeitsprozess einordnen lässt (vgl. Braverman 1974, 57; Friedman 1977, 78; Berger 1999, 155).

In der Forschung wird dieses Problem aus unterschiedlichen theoretischen Perspektiven beschrieben. Marxisten sprechen davon, dass der Einkauf von Arbeitskraft durch den Kapitalisten nicht gleichbedeutend mit der realen Nutzung der Arbeitskraft durch das Kapital ist (vgl. Marx 1962, 532 f.). Systemtheoretiker reden von dem Problem, dass die Mitgliedschaftsmotivation - also die Motivation zum Eintritt und Verbleib in der Organisation - nicht automatisch auch zu einer Leistungsmotivation - also zur Motivation zur Erbringung der von der Organisation erwarteten Leistung - führt (Luhmann 1975, 104 ff.). Und Institutionenökonomen sprechen von dem Problem, dass der Prinzipal an der Spitze der Organisation nur begrenzt in der Lage ist, sicherzustellen, dass die mit Geld eingekauften Agenten auch faktisch im Sinne der Organisation tätig werden (Saam 2002, 28 ff.).

In Organisationen wird deswegen ein hohes Maß an Kreativität entwickelt, um dieses Problem der Übersetzung von Mitgliedschaftsmotivation in Leistungsmotivation in den Griff zu bekommen. Mitarbeiter am Fließband bekommen Akkordprämien, wenn sie überdurchschnittliche Leistungen erbracht haben. Vertriebsmitarbeiter werden belohnt, wenn sie überdurchschnittlich viele Produkte verkaufen. Professoren bekommen im Rahmen von leistungsorientierter Mittelzuweisung für jeden publizierten Artikel oder jedes eingeworbene Drittmittelprojekt eine kleine finanzielle Belohnung. Die Vorstellung ist, dass man die Arbeits- 
kraft von Mitarbeitern besser nutzen kann, wenn man diese nicht (nur) pauschal bezahlt, sondern (zusätzlich) für genau definierte Leistungen entlohnt.

Die Grenzen dieser formalisierten Leistungsanreize werden jedoch schnell deutlich. Die Kriterien zur Erreichung von Leistungsprämien müssen im Voraus genau definiert werden. Das Leistungssystem muss so weit verallgemeinert und objektiviert werden, dass alle Mitarbeiter Zugang zu diesen Prämien haben. Und häufig ist auch die Zustimmung der Mitarbeitervertretung zu diesen Prämienzahlungen nötig. Das System der Leistungsanreize wird dadurch zwangsläufig so statisch, dass es sich bestenfalls für hochstandardisierte Arbeitsprozesse wie die Arbeit am Fließband, den Verkauf von Produkten oder - so jedenfalls die Vorstellung einiger Universitätsleitungen - für das Schreiben von wissenschaftlichen Fachartikeln eignet. ${ }^{18}$

Wie lassen sich die Nachteile solcher formalisierten Leistungsanreizsysteme vermeiden?

\subsection{Subtile Belohnungsmechanismen}

Eine Möglichkeit besteht darin, informale Belohnungssysteme für besondere Leistungen der Mitarbeiter zuzulassen. Wir kennen unterschiedlichste Organisationen, in denen informale Belohnungsmechanismen von Vorgesetzten nicht nur geduldet, sondern häufig sogar befördert werden. De jure sind dies arbeits- und strafrechtlich relevante Formen von Unterschlagung, Bestechung, Betrug oder Vergewaltigung, de facto gleichzeitig aber auch nicht selten effiziente informale Gratifikationssysteme der Organisation (Dalton 1959, 208).

Eine erste Variante für diese informalen Belohnungssysteme besteht in der kontrollierten Duldung von Unterschlagungen und Diebstahl. Man denke an die teilweise ausgeklügelten Systeme, mit denen in Häfen, Hospitälern oder Kaufhäusern Produkte als beschädigt deklariert werden, damit diese günstig oder kostenlos von besonders verdienten Mitarbeitern erworben werden können. Man denke an die Kellner in Restaurants, ihre kleine Tricksereien an der Kasse, die

18 Aber schon bei der Belohnung des Publizierens von Fachartikeln oder des Einwerbens von Drittmitteln werden die Grenzen deutlich. In den Steuerungsvorstellungen einiger Hochschulpolitiker funktionieren Wissenschaftler nach einem aus der Fließbandarbeit abgeleiteten simplen Kausalmodell. Wenn man Geldprämien für das Schreiben von Fachartikeln oder das Einwerben von Drittmitteln zur Verfügung stellt, werden Wissenschaftler, so die Annahme, zusätzliche Anstrengungen in diese Richtung unternehmen. Die negativen Auswirkungen dieser Motivationsmaßnahmen sind unter dem Begriff des „Crowding-out“ - der Motivationsverdrängung - ausführlich beschrieben worden. 
von ihnen - aber nicht selten auch von ihren Vorgesetzten - als Teil ihres Lohns betrachtet werden. Oder man denke an Zeitungsredaktionen, in denen lange Zeit die Regel galt, dass eine „gute Geschichte“ durch besonders großzügige Aufwandsentschädigungen belohnt wird. ${ }^{19}$

Eine zweite Variante subtiler Belohnungen ist die Duldung von verschiedenen Formen von Korruption. Das kann die geduldete Aneignung von Bestechungsgeldern, die Erwartung größerer Geschenke oder die Duldung der Annahme sexueller Dienstleistungen sein. So wissen wir von vielen Organisationen, dass Vorgesetzte in begrenztem Rahmen die Annahme kleinerer Annehmlichkeiten von Kunden oder Zulieferern dulden oder dass Kommandeure trotz ausdrücklichen Verbotes der obersten Heeresleitung die sexuellen Beziehungen von Soldaten zu Einheimischen dulden, wenn sie den Eindruck haben, dass Erstere bei der Tötung von gegnerischen Soldaten - oder auch Zivilisten - ihr Soll übererfüllen (vgl. zum extremeren Fall der Duldung sexueller Gewalttaten von deutschen Soldaten in der Sowjetunion Mühlhäuser 2010).

Eine dritte Variante ist die Duldung von Abwesenheiten. Unter Bergarbeitern bildet sich die Erwartung aus, dass in der Zeit unter Tage hart angepackt wird auch über die formalen Vorgaben hinaus -, dass man sich nach drei oder vier Tagen harten Arbeitens dann aber auch einen eigentlich nicht vorgesehenen freien Tag genehmigen kann, an dem man sich gemeinsam betrinkt (vgl. Gouldner 1954). Wir wissen, dass Unternehmen, die verdienten Mitarbeitern aufgrund von konzernweiten Richtlinien nicht mehr bezahlen können, diesen freie Arbeitstage zugestehen, um sie nicht als Mitarbeiter zu verlieren (Dalton 1959, 204).

\section{Der Charme informaler Entlohnungen in Organisationen}

Man fragt sich, warum man die Belohnungsstruktur nicht einfach formalisiert. Gerade an der Spitze der Organisation kann man ja erkennen, dass sich Organisationsmitglieder vielfältige Privilegien nicht nur in Form von hohen Gehältern, sondern auch durch die Nutzung firmeneigener Fahrzeuge für private Zwecke,

19 Siehe dazu die Studien zum Phänomen des „knock-off“ im Hotel- und Gaststättengewerbe. Siehe z.B. Mars u.a. (1979, 33-34). Mars/Nicod $(1984,8)$ berechnen das „total rewards system“ im Hotel- und Gaststättengewerbe als Summe aus „basic pay + subsidised lodging + subsidised or free food + tips + fiddles + ,knock-offs““. Siehe zu den informalen Belohnungen in Zeitungsredaktionen das Motto unter Journalisten „A good story deserves good expenses“ (Mars 2013, 39). 
durch firmenfinanzierte Freizeitmöglichkeiten für die ganze Familie, Zugang zu Sport-Events usw. auf legalem Wege zugestehen. Was läge also näher, als für alle Mitglieder die vielfältigen informalen Belohnungsmechanismen zu formalisieren?

\subsection{Gründe für informale Belohnungen}

Ein erster Grund für die Beibehaltung informaler Entlohnung ist der, dass eine Beförderung oder eine höhere Entlohnung häufig formal nicht durchsetzbar sind (Dalton 1959, 198). In vielen Organisationen sind wegen des pyramidenartigen Aufbaus die Aufstiegsmöglichkeiten begrenzt. Es stehen nicht ausreichend Stellen zur Verfügung, um Leistungsträger zu befördern. Gleichzeitig sind aber auch die Möglichkeiten, Gehälter für Personen auf einer bestimmten Hierarchiestufe zu erhöhen, begrenzt. In solchen Fällen sind informale Formen von Belohnung eine Möglichkeit, diese Leistungsträger nicht zu verlieren.

Ein zweiter Grund liegt darin, dass Organisationsmitglieder für besonders unattraktive Tätigkeiten motiviert werden müssen (Dalton 1959, 198). In jeder Organisation gibt es Aufgaben, für die nur schwer Personal zu finden ist. Man denke nur an Tätigkeiten in der für den Rostschutz wichtigen Galvanik in der Automobilzulieferindustrie, an die Reinigungstätigkeiten in Bussen und Zügen oder an die Löschung von Kinderpornografie bei den Internetbetreibern von sozialen Netzwerken. Hier bilden sich nicht selten inoffizielle Deals aus, dass Mitarbeiter Werkzeuge für private Zwecke verwenden können, dass sie sich von Kunden liegengelassene Gegenstände aneignen dürfen oder sich formal nicht erlaubte Arbeitserleichterungen herausnehmen können, wenn sie nur die Aufgaben zur Zufriedenheit der Organisation erledigen.

Manchmal geht es dabei drittens gar nicht so sehr nur um kleinere illegale Vergünstigungen und Erleichterungen, sondern um die Anreicherung der Arbeit mit zusätzlicher Spannung. Wenn Mitarbeiter anfangen, sich bei der Arbeit zu langweilen, nutzen sie kleinere Diebstähle, das Erschleichen von Vorteilen im Kundenkontakt und das Erschummeln von Arbeitserleichterungen dazu, ihre Arbeit wieder interessanter zu machen. Durch das Tun von Verbotenem bekommt die Arbeit einen Thrill, den Lawrence R. Zeitlin (1971, 24) als eine Art selbstorganisierter Arbeitsanreicherung bezeichnet - ,Job Enrichment“ -, wodurch die Mitarbeiter die Arbeit wieder mit Sinn anreichern können. ${ }^{20}$

20 Die These von Zeitlin basiert auf der Untersuchung der Motive von Diebstählen durch Mitarbeiter von Kleidungsgeschäften. Die Überlegungen ähneln stark dem von Michael Burawoy 
Viertens wird informale Entlohnung eingesetzt, wenn Mitglieder für offiziell verbotenes Verhalten motiviert werden müssen. Man denke beispielsweise an Fälle, in denen der Vorstand mit kreativen Finanzinstrumenten wie den berüchtigten Special Purpose Entities die Bilanzen fälscht und sich für diese Leistung abgesegnet durch die Wirtschaftsprüfungsgesellschaft - als Geschäftsführer der Entities zusätzlich Millionen auszahlen lässt. ${ }^{21}$ Mitglieder eines Organisationskomitees für eine Fußballweltmeisterschaft bekommen dann Provisionen in der Höhe von mehreren Hunderttausend Euro nicht nur für die Vermittlung von rechtlich legalen Sponsoring-, Werbe- und Finanzierungsverträgen, sondern besonders auch für den illegalen Stimmenkauf bei Fußballfunktionären. Oder man denke an die SS-Männer, Ordnungspolizisten oder Wehrmachtssoldaten, die sich während der NS-Zeit an ihren jüdischen Opfern bereichert haben, was zwar offiziell bei Todesstrafe verboten war, aber noch im Graubereich des Erlaubten lag und von ihren Vorgesetzten geduldet wurde, weil diese Tötungsaufgaben als belastend definiert waren. Solche Formen von brauchbarer Illegalität können allein schon deswegen nicht offiziell entlohnt werden, weil dadurch die illegalen Praktiken bekannt werden würden (Dalton 1959, 215).

\subsection{Vorteile informaler Belohnungen}

Informale Belohnungen bringen offensichtliche Vorteile mit sich. Von ihrer formalen Struktur her sind Organisationen „tauschfeindlich“ gebaut. Mitarbeiter werden von Organisationen in der Regel durch einen Pauschallohn vergütet und können nicht erwarten, dass sie für jede Handlung noch zusätzlich von Kollegen, Vorgesetzten oder Untergebenen be- oder entlohnt werden (vgl. Luhmann 1964, 288 ff.). Ein Kollege, der eine Information nicht wie formal vorgeschrieben an eine Kollegin weitergibt, sondern die Informationsweitergabe gern als persönlichen Gefallen wahrgenommen sehen möchte, würde bei der Kollegin Irritationen hervorrufen. Ein Sekretär, der von seiner Chefin erwartet, dass das Tippen jedes Briefes mit einem mehr als symbolischen Dankeschön - Pralinen und Blumen, Sonderurlaub oder verlängerte Pausen - belohnt wird, hätte mittelfristig Schwierigkeiten, sich in einer Organisation zu halten.

(1979) beobachteten Konzept des „making out“ auf dem shop floor. Zu den Problemen der Fragen nach Motiven siehe Mills (1940); siehe auch ausführlich Gerth/Mills (1954).

21 Zum Fall der sehr gut untersuchten Special Purpose Entities siehe Barreveld (2002); Fox (2004); Fusaro/Miller (2002); McLean/Elkind (2004); Salter (2008). 
Während die Organisationen von ihrer Formalstruktur her „tauschfeindlich“ gebaut sind, spielt Tausch bei der Durchsetzung informeller Erwartungen eine zentrale Rolle. In Organisationen findet dieser Tausch selten unmittelbar statt. Direkte Deals etwa in der Form „Du erlaubst mir jetzt das Rauchen in meinem Büro, dafür bleibe ich heute länger" sind eher die Ausnahme. Stattdessen wird davon ausgegangen, dass sich das informelle Entgegenkommen gegenüber einem Kollegen, einem Vorgesetzten oder einem Untergebenen später schon auszahlen wird (vgl. Luhmann 2002, 44). Man pflastert den kleinen Dienstweg mit Gefälligkeiten und hofft, dass der andere seinen Teil zur Wegepflege beiträgt. Die Duldung von Unterschlagungen, Korruption und Arbeitserleichterungen ist eine billige Möglichkeit, die Loyalität von Organisationsmitgliedern zu erhalten (Martin 1962, 115). ${ }^{22}$

Letztlich ist dies natürlich für die Seite, die in Vorleistung geht, riskant. Man kann nie sicher sein, ob das eigene Entgegenkommen auch ein Entgegenkommen des Gegenübers zur Folge hat. Wenn eine Mitarbeiterin bereit ist, bei wichtigen Aufträgen auch einmal über die gesetzliche Arbeitszeit hinaus in der Firma zu bleiben, dann kann sie hoffen, dass ihre Chefin beim gelegentlichen Zuspätkommen am Morgen ein Auge zudrückt. Sicher sein kann sie sich nicht. Wenn der Generalsekretär einer Partei beim Bekanntwerden von illegalen Parteispenden für seinen Parteivorsitzenden den Kopf hinhält und zurücktritt, dann kann er darauf hoffen, dass er später durch einen Posten als Verteidigungs- oder Arbeitsminister belohnt wird, sicher sein kann er sich aber nicht. Diese Form von Leistungen, bei denen es keine Sicherheit gibt, dass sie auch entgolten werden, basiert auf einer Einstellung, die in der Informalität eine wichtige Rolle spielt: Vertrauen (vgl. dazu theoretisch immer noch maßgeblich Luhmann 1968, 48 ff.).

\section{Die Grenzen informaler Belohnungssysteme}

Das Problem informaler Belohnungen besteht darin, dass alle Mitarbeiter sie in Anspruch nehmen möchten. In fast jeder Organisation gibt es die Tendenz, dass sich Mitarbeiter im Vergleich zu anderen schlechter behandelt fühlen. Wird jetzt beobachtet, dass andere Mitarbeiter durch Aneignung von Materialien, Entgegennahme von Geschenken oder die Schonung ihrer Arbeitskraft Vorteile erlan-

22 Im Original heißt es bei J. P. Martin (1962, 115): ,a cheap way of buying good morale and personal relationship“. 
gen, wächst das Verlangen nach vergleichbarer Belohnung. Aus diesen Gründen versuchen Organisationen, informale Belohnungssysteme einzuregulieren.

\subsection{Sensibilitäten}

Eine erste Form der Einregulierung ist eine Generalisierung der informalen Belohnung für alle Mitarbeiter. Es fällt auf, wie sensibel in Organisationen darauf geachtet wird, dass informale Belohnung nicht das Privileg weniger in der Organisation gut positionierter Mitarbeiter bleibt, sondern dass diese allen Leistungsträgern einer Organisation zugutekommt. Nicht selten gibt es in Organisationen Mechanismen, mit denen die informalen Belohnungen, die Mitarbeiter im Außendienst erzielen, mit den Mitarbeitern im Innendienst geteilt werden. Durch diese häufig fein austarierte Verteilung von informalen Vorteilen wird sichergestellt, dass Mitarbeiter die informale Belohnung nicht als unfair empfinden.

Eine zweite Form der Einregulierung besteht in einem hohen Maß an Sensibilität dafür, auf wessen Kosten man sich individuelle Vorteile verschafft (siehe zur Problematik Robinson/Bennett 1995). Studien über Regelabweichungen in Restaurants, im Einzelhandel und in Krankenhäusern haben gezeigt, dass sehr genau unterschieden wird, ob sich Mitarbeiter auf Kosten der Organisation, auf Kosten der Kunden oder auf Kosten der anderen Mitarbeiter bereichern (siehe dazu z.B. Martin 1962, 82 f.; Mars 1973, 201; Hawkins 1984, 48 f.). Am wenigsten scheint akzeptiert zu sein, wenn sich Mitarbeiter auf Kosten anderer Mitarbeiter bereichern - zum Beispiel in Form von Tricksereien bei der Verteilung von Prämien oder Trinkgeldern oder beim Diebstahl von Eigentum anderer (siehe dazu Horning 1970). Die persönliche Bereicherung durch Mitnahme von Organisationseigentum, Nutzung von Werkzeugen für private Zwecke oder Manipulation von Reisekostenabrechnungen wird noch am ehesten toleriert. Die Bereicherung auf Kosten von Kunden und Klienten zum Beispiel in Form des Ausstellens überhöhter Rechnungen, von Tricksereien bei der Rückgabe von Restgeld oder Diebstahl von Eigentum wird als wenig legitim betrachtet, scheint aber Akzeptanz zu finden, wenn sich die Mitarbeiter in ihrer Leistung nicht ausreichend gewürdigt fühlen.

Eine dritte Form der Einregulierung ist die informale Verständigung über die Form illegaler Belohnungen. So wird im Hotel- und Gaststättengewerbe akzeptiert, dass man bei der Erstellung von Rechnungen und der Rückgabe von Quittungen trickst, aber das Bestehlen des Gastes gilt als Tabu (Mars 1973, 201). Bei der Aneignung von Waren durch Entladeteams am Hafen gilt es als legitim, sich bei für den Verkauf bestimmten Gütern wie Whisky, Armbanduhren oder Radios 
zu bedienen, aber der Diebstahl von persönlichem Gepäck gilt als illegitim (Mars 1974, 224).

\subsection{Die Rolle von Kollegen, Vorgesetzten und Untergebenen}

Genauso wie einzelne Mitarbeiter die ihnen formal zustehenden Gehälter, ihre offiziellen Arbeitszeiten und ihre amtlichen Stellenbeschreibungen nicht selbst festlegen, können sie auch ihre informalen Belohnungen, inoffiziellen Arbeitszeiten und ihre faktischen Arbeitsanforderungen nicht selbst festlegen. Sicherlich - es gibt Unterschiede. Die formalen Entlohnungen, die offiziellen Arbeitszeiten und amtlichen Stellenbeschreibungen werden durch Entscheidungen festgelegt, können in den Akten nachgelesen werden und vor Gericht eingeklagt werden. Informale Entlohnungen, inoffizielle Arbeitszeiten und faktische Arbeitserleichterungen spielen sich dagegen eher langsam ein, werden nicht in den Akten festgehalten und können auch nicht vor Gericht durchgesetzt werden. Aber in beiden Fällen handelt es sich um die Ausbildung von Erwartungen, die nicht von einzelnen Mitarbeitern selbst festgelegt werden können.

Bei der Einregulierung informaler Entlohnung nehmen die unmittelbaren Kollegen eine wichtige Rolle ein. Sie achten darauf, dass sich die Verschaffung von Vorteilen an generalisierten Gerechtigkeitsnormen orientiert. Aber auch Vorgesetzte spielen bei der Einregulierung eine wichtige Rolle, weil sie über mehr oder minder subtile Zeichen signalisieren, wo die Grenzen informaler Belohnung liegen. Selbst Untergebene können bei der Einregulierung eine wichtige Bedeutung haben, weil Vorgesetzte wissen, dass ihr formal nicht korrektes Verhalten durch diese beobachtet und gemeldet werden könnte.

Der Effekt dieser gegenseitigen Beobachtung besteht darin, dass eine Sensibilität dafür entsteht, dass informale Belohnungen mit Leistungen im Sinne der Organisation verknüpft bleiben. So kann sichergestellt werden, dass die Aneignung von Materialien, die Entgegennahme von kleinen Vergünstigungen von Kunden oder das Zugestehen von formal nicht vorgesehenen Arbeitserleichterungen den Mitarbeitern zugutekommen, die sich durch eine besondere Leistungsbereitschaft hervorgetan haben. Schließlich macht es für eine Organisation einen zentralen Unterschied, ob beispielsweise Polizisten einen kleinen Teil des bei einer Razzia beschlagnahmten Drogengelds von Kriminellen als Prämie für besonderes Engagement an sich nehmen oder ob sie sich mit Drogengeld von Kriminellen bestechen lassen und deshalb eine Razzia nicht durchführen. 


\section{Fazit: Unterbindung oder Duldung}

Die Risiken informaler Belohnung dürfen jedoch nicht übersehen werden. Informale Belohnungen können Bestandteil der normalen Lohnerwartung von Organisationsmitgliedern werden (siehe dazu Dalton 1959, 213). In der Organisation droht das Verständnis dafür verloren zu gehen, dass die Duldung von Unterschlagungen, milde Formen von Korruption oder punktuelle Arbeitsbefreiung Mittel zur Motivation für besondere Leistungen sind. Die informale Entlohnung, die eigentlich verbotene Prämienzahlung durch Kooperationspartner oder eine vertraglich nicht vorgesehene punktuelle Arbeitsbefreiung werden dann unabhängig von besonderen Leistungen für die Organisation in Anspruch genommen.

Die Mechanismen informaler Belohnung bringen die üblichen Nachteile illegaler Märkte mit sich (siehe dazu Schmidt/Garschagen 1978, 567 f.). Wegen des „Untergrundcharakters“ des informalen Belohnungssystems herrscht ein hohes Maß an Intransparenz. Der Umfang des informalen Belohnungssystems kann weder systematisch erhoben noch offen diskutiert werden. Wegen der Intransparenz besteht die Gefahr, dass das informale Belohnungssystem immer mehr zu einem Verständnis der Organisation als Selbstbedienungsladen führt.

Daher liegt es nahe, dass die Organisationen auf Unterschlagungen, Korruption und Arbeitsverweigerung mit einer Unterbindung des informalen Belohnungssystems reagieren und die Mitglieder mit Abmahnung, Kündigung und strafrechtlicher Verfolgung sanktionieren. Die Praxis in Organisationen scheint jedoch häufig eine andere zu sein: Die Erzielung persönlicher Vorteile wird in einem allgemein geteilten Rahmen geduldet (siehe dazu Henry 1978).

Vielleicht steckt hinter dieser Duldung ein gewisses Maß an organisatorischer Weisheit, weil die Organisation dadurch Flexibilitätsvorteile im Belohnungssystem erlangt. ${ }^{23}$ Weil es sich bei Unterschlagungen, Korruption und Arbeitserleichterungen um Privilegien handelt, die den Mitarbeitern laut Arbeitsvertrag formal nicht zustehen, können besonders Vorgesetzte, aber in letzter Konsequenz auch Untergebene die Inanspruchnahme dieser informalen Belohnungen jederzeit unterbinden, wenn die Leistungen nicht mehr stimmen. Weitergehend können sie sogar die Inanspruchnahme von Privilegien als Form nega-

23 Lawrence R. Zeitlin $(1971,26)$ schlägt vor, folgende Kalkulation vorzunehmen: „1. How much is employee theft actually costing us? 2. What increase in employee dissatisfaction could we expect if we controlled theft? 3. What increase in employee turn-over could we expect? 4 . What would it cost to build employee motivation up to a desirable level by conventional means of job enrichment or through higher salaries?“ 
tiver Sanktionierung nutzen, indem sie Mitarbeiter wegen Unterschlagung, Korruption oder Leistungsverweigerung arbeits- oder strafrechtlich belangen.

Anmerkung und Danksagung: Bei diesem Artikel handelt es sich um erste Überlegungen zu einem Kapitel in meinem geplanten Buch über brauchbare und unbrauchbare Illegalität in Organisationen. Ich danke Finn-Rasmus Bull, Markus Jüttner, Sven Kette, Tabea Koepp und Judith Muster sowie den Herausgebern der Zeitschrift ARBEIT und zwei anonymen Gutachtern für hilfreiche Kommentare zu einer ersten Fassung des Artikels.

\section{Literatur}

Abagnale Jr., Frank W., Stan Redding (2000): Catch Me If You Can. The amazing true story of the youngest and most daring con man in the history of fun and profit. New York: Broadway Books

Barker, Thomas, David L. Carter (Hg.) (1994): Police Deviance. London: Routledge

Barreveld, Dirk J. (2002): The ENRON Collapse. Creative accounting, wrong economics or criminal acts? San Jose: Writers Club Press

Bensman, Joseph, Israel Gerver (1963): Crime and Punishment in the Factory. The function of deviancy in maintaining the social system; in: American Sociological Review, 28, 588-598

Berger, Johannes (1999): Der Konsensbedarf der Wirtschaft; in: Johannes Berger (Hg.): Die Wirtschaft der modernen Gesellschaft. Frankfurt a.M., New York: Campus, 155-194

Blauner, Robert (1964): Alienation and Freedom. The factory worker and his industry. Chicago: University of Chicago Press

Bliss, Edwin C., Isamu S. Aoki (1993): Are your employees stealing you blind?. Amsterdam, San Diego: Pfeiffer

Braverman, Harry (1974): Labor and Monopoly Capital. The Degradation of Work in the Twentieth Century. New York, London: Monthly Review Press

Bryant, Donald T. (1975): A Manager's Guide to Withdrawal from Work. Brighton: Institute of Manpower Studies

Burawoy, Michael (1979): Manufacturing Consent. Chicago, London: University of Chicago Press

Calavita, Kitty, Henry N. Pontell (1993): Savings and Loan Fraud as Organized Crime. Toward a conceptual typology of corporate illegality; in: Criminology, 31, 519-548

Chambliss, William J. (1989): State-organized Crime; in: Criminology, 27, 183-208

Clark, John P., Richard C. Hollinger (1983): Theft by Employees in Work Organizations. New York: Lexington Books

Clinard, Marshall Barron, Richard Quinney (Hg.) (1973): Criminal Behavior Systems. A Typology. New York: Holt Rinehart and Winston

Coleman, James W. (1987): Toward an integrated theory of white-collar crime; in: American Journal of Sociology, 93, 406-439 
Dabney, Dean (1995): Neutralization and Deviance in the Workplace. Theft of supplies in medicines by hospital nurses; in: Deviant Behavior, 16, 313-331

Dalton, Melville (1959): Men Who Manage. New York: Wiley

Edelhertz, Herbert (1970): The Nature, Impact, and Prosecution of White Collar Crime. Washington DC: U.S. Government Printing Office

Fox, Loren (2004): Enron. The Rise and Fall. Hoboken: Wiley

Friedman, Andrew (1977): Industry and Labour. London: Macmillan

Friedrichs, David 0. (2010): Trusted Criminals. New York: Wadsworth

Fusaro, Peter C., Ross M. Miller (2002): What Went Wrong At Enron. Everyone's guide to the largest bankruptcy in U. S. history. Hoboken: Wiley

Gambetta, Diego (2006): Can We Make Sense of Suicide Missions?; in: Diego Gambetta (Hg.): Making Sense of Suicide Missions. Oxford: Oxford University Press, 259-300

Gerth, Hans, C. Wright Mills (1954): Character and Social Structure. London: Routledge \& Kegan Paul

Gouldner, Alvin W. (1954): Wild Cat Strike. New York: Harper

Hašek, Jaroslav (1960): Die Abenteuer des braven Soldaten Schwejk. Band 1. Reinbek: Rowohlt Hawkins, Richard (1984): Employee Theft in the Restaurant Trade. Forms of ripping off by waiters at work; in: Deviant Behavior, 5, 47-69

Henry, Stuart (1978): The Hidden Economy. The context and control of borderline crime. London: Martin Robertson

Henry, Stuart, Gerald Mars (1978): Crime at Work: The social construction of amateur property theft; in: Sociology, 12, 245-263

Höffling, Christian (2002): Korruption als soziale Beziehung. Opladen: Leske \& Budrich

Höhn, Reinhard (1986): Die innere Kündigung im Unternehmen. Bad Harzburg: WWT

Höhn, Reinhard (1989): Die innere Kündigung in der öffentlichen Verwaltung. Ursachen, Folgen, Gegenmaßnahmen. Stuttgart: Moll

Hollinger, Richard C., John P. Clark (1982): Employee Deviance. A response to the perceived quality of the work experience; in: Work and Occupation, 9, 97-114

Horning, Donald N. M. (1970): Blue-Collar Theft. Conception of property, attitudes toward pilfering, and work group norms; in: Erwin O. Smigel, H. Laurence Ross (Hg.): Crimes Against Bureaucracy. New York: Van Nostrand, 46-64

Kühl, Stefan (2011): Organisationen. Eine sehr kurze Einführung. Wiesbaden: VS

Kühl, Stefan (2017): Arbeit - Marxistische und systemtheoretische Zugänge. Wiesbaden: Springer VS

Lauderdale, Pat, Harold Grasmick, John P. Clark (1978): Corporate Environments, Corporate Crime, and Deterrence; in: Marvin D. Krohn, Ronald L. Akers (Hg.): Crime, Law, and Sanctions. Beverly Hills, London: Sage, 137-158

Luhmann, Niklas (1964): Funktionen und Folgen formaler Organisation. Berlin: Duncker \& Humblot

Luhmann, Niklas (1968): Vertrauen. Stuttgart: Lucius \& Lucius

Luhmann, Niklas (1975): Macht. Stuttgart: Enke

Luhmann, Niklas (2002): Die Politik der Gesellschaft. Frankfurt a. M.: Suhrkamp

Mangione, Thomas, Robert Quinn (1975): Job Satisfaction, Counterproductive Behavior, and Drug Use at Work; in: Journal of Applied Psychology, 60, 114-116

Mars, Gerald (1973): Hotel Pilferage. A case study in occupational theft; in: Malcolm Warner (Hg.): The Sociology of the Workplace. New York: Halsted, 200-210 
Mars, Gerald (1974): Dock Pilferage. A case study in occupational theft; in: Paul Rock, Mary McIntosh (Hg.): Deviance and Social Control. London: Tavistock, 209-228

Mars, Gerald (1982): Cheats at Work. An anthropology of the workplace. London: Routledge Mars, Gerald (2013): Locating Deviance. Crime, change and organizations. Farnham, Surrey: Ashgate

Mars, Gerald, Donald T. Bryant, Peter Mitchell (1979): Manpower Problems in the Hotel and Catering Industry. Farnborough: Saxon House

Mars, Gerald, Michael Nicod (1984): The World of Waiters. London: Allen \& Unwin

Martin, J. Patrick (1962): Offenders As Employees. New York: St. Martin's Press

Marx, Karl (1962): Das Kapital. Erstes Buch. Marx-Engels-Werke, Band 23. Berlin: Dietz

McLean, Bethany, Peter Elkind (2004): The Smartest Guys in the Room. The amazing rise and scandalous fall of Enron. New York: Portfolio

Michalowski, Raymond J. (1985): Order, Law and Crime. New York: Random House

Miller, Nathan (1992): Stealing from America. A History of Corruption from Jamestown to Reagan. New York: Paragon House

Mills, C. Wright (1940): Situated Actions and Vocabularies of Motive; in: American Sociological Review, 5, 904-913

Morris, Albert (1934): Criminology. New York: Longman

Mühlhäuser, Regina (2010): Eroberungen. Sexuelle Gewalttaten und intime Beziehungen deutscher Soldaten in der Sowjetunion 1941-1945. Hamburg: Hamburger Edition

Neckel, Sighard (1995): Der unmoralische Tausch. Eine Soziologie der Käuflichkeit; in: Kursbuch, 120, 9-16

New, Robert Cameron (1994): Are Your Employees Stealing You Blind? Answers and solutions for retailers and other small businesses. Amherst: HRD Press

Pinto, Jonathan, Carrie R. Leana, Frits K. Pil (2008): Corrupt Organizations or Organizations of Corrupt Individuals? Two Types of Organization-Level Corruption; in: Academy of Management Review, 33, 685-709

Punch, Maurice (1996): Dirty Business. Exploring corporate misconduct. London, Thousand Oaks, New Delhi: Sage

Robinson, Sandra L., Rebecca J. Bennett (1995): A Typology of Deviant Workplace Behaviors. A multidimensional scaling study; in: Academy of Management Journal, 38, 555-572

Saam, Nicole J. (2002): Prinzipale, Agenten und Macht. Eine machttheoretische Erweiterung der Agenturtheorie und ihre Anwendung auf Interaktionsstrukturen in der Organisationsberatung. Tübingen: J. C. B. Mohr

Sallaz, Jeffrey J. (2009): The Labor of Luck. Casino capitalism in the United States and South Africa. Berkeley: California University Press

Salter, Malcolm S. (2008): Innovation Corrupted. The origins and legacy of Enron's collapse. Cambridge: Harvard University Press

Schmidt, Karl, Christine Garschagen (1978): Korruption; in: Willi Albers, Karl Erich Born, Ernst Dürr, Helmut Hesse, Alfons Kraft, Heinz Lampert, Klaus Rose, Hans-Heinrich Rupp, Harald Scherf, Kurt Schmidt, Wittman Waldemar (Hg.): Handwörterbuch der Wirtschaftswissenschaft. Stuttgart, New York, Tübingen, Göttingen, Zürich: Gustav Fischer, J. C. B. Mohr, Vandenhoeck \& Ruprecht, 565-573

Senturia, Joseph J. (1931): Corruption, Political (General); in: Edwin R. A. Seligman, Alvin Johnson (Hg.): Encyclopedia of the Social Science. Band 4. New York, 448-452

Smigel, Erwin O., Laurence H. Ross (1970): Introduction; in: Erwin O. Smigel, H. Laurence Ross (Hg.): Crimes Against Bureaucracy. New York: Van Nostrand, 1-14 
Sutherland, Edwin H. (1949): White Collar Crime. New York: Holt, Rinehart \& Winston

Sutherland, Edwin H. (1983): White-Collar Crime. The uncut version. London: Yale University Press

Tucker, James (1989): Employee Theft as Social Control; in: Deviant Behavior, 10, 319-334

Van Natta, Don, Neela Benrjee (2002): Top G.O.P. Donors in Industry Met Cheney Panel; in: New York Times, 1. März

Wardi, Yoav, Ely Weitz (2004): Misbehavior in Organizations. Theory, research, and management. Mahwah, NJ: Erlbaum

Wheeler, Stanton, Mitchell Lewis Rothman (1982): The Organization as Weapon in White-Collar Crime; in: Michigan Law Review, 80, 7, 1403-1426

Zeitlin, Lawrence R. (1971): A Little Larceny Can Do a Lot for Employee Morale; in: Psychology Today, 5, 22-64

Zuckmayer, Carl (2004): Der Hauptmann von Köpenick. Ein deutsches Märchen in drei Akten. Frankfurt a. M.: Fischer 